\title{
Preoperative skin antisepsis with chlorhexidine gluconate versus povidone-iodine: a prospective analysis of 6959 consecutive spinal surgery patients
}

\author{
George M. Ghobrial, MD, Michael Y. Wang, MD, Barth A. Green, MD, Howard B. Levene, MD, PhD, \\ Glen Manzano, MD, Steven Vanni, DO, DC, Robert M. Starke, MD, MSc, \\ George Jimsheleishvili, MD, Kenneth M. Crandall, MD, Marina Dididze, MD, PhD, and \\ Allan D. Levi, MD, PhD
}

Department of Neurological Surgery and The Miami Project to Cure Paralysis, University of Miami Miller School of Medicine, Miami, Florida

OBJECTIVE The aim of this study was to determine the efficacy of 2 common preoperative surgical skin antiseptic agents, ChloraPrep and Betadine, in the reduction of postoperative surgical site infection (SSI) in spinal surgery procedures.

\begin{abstract}
METHODS Two preoperative surgical skin antiseptic agents-ChloraPrep ( $2 \%$ chlorhexidine gluconate and $70 \%$ isopropyl alcohol) and Betadine (7.5\% povidone-iodine solution)-were prospectively compared across 2 consecutive time periods for all consecutive adult neurosurgical spine patients. The primary end point was the incidence of SSI.

RESULTS A total of 6959 consecutive spinal surgery patients were identified from July 1, 2011, through August 31, 2015, with $4495(64.6 \%)$ and 2464 (35.4\%) patients treated at facilities 1 and 2, respectively. Sixty-nine (0.992\%) SSIs were observed. There was no significant difference in the incidence of infection between patients prepared with Betadine (33 [1.036\%] of 3185) and those prepared with ChloraPrep (36 [0.954\%] of 3774; $p=0.728)$. Neither was there a significant difference in the incidence of infection in the patients treated at facility 1 (52 [1.157\%] of 4495) versus facility $2(17[0.690 \%]$ of $2464 ; p=0.06)$. Among the patients with SSI, the most common indication was degenerative disease (48 [69.6\%] of 69). Fifty-one (74\%) patients with SSI had undergone instrumented fusions in the index operation, and 38 $(55 \%)$ patients with SSI had undergone revision surgeries. The incidence of SSI for minimally invasive and open surgery was $0.226 \%$ ( 2 of 885 cases) and $1.103 \%$ (67 of 6074 cases), respectively.
\end{abstract}

CONCLUSIONS The choice of either ChloraPrep or Betadine for preoperative skin antisepsis in spinal surgery had no significant impact on the incidence of postoperative SSI.

https://thejns.org/doi/abs/10.3171/2017.5.SPINE17158

KEY WORDS skin antisepsis; antiseptic solutions; chlorhexidine; povidone-iodine; spinal surgery; surgical site infection; ChloraPrep; DuraPrep

$\mathrm{C}$ OST-CONTAINMENT measures that can be instituted in the clinical setting and have an immediate immedicine, as evideced by the gradual incorporation of value-based care initiatives. ${ }^{20}$ Postoperative surgical site infection (SSI) persists as the most common hospital-acquired infection in general surgery departments, as well as a common problem in the spine population because of varying surgical invasiveness, patient comorbidities, and antiseptic measures, with an incidence ranging from $0 \%$ to $16 \% .{ }^{25}$ This area of study is particularly relevant as SSIs are linked to higher costs, ,,9,12 poor patient outcomes, morbidity, and mortality. ${ }^{12,35}$

Guidance in the literature for spinal surgeons is limited regarding the ideal preoperative skin antisepsis. Dissimilar study populations, SSI definitions, surgical procedures,

ABBREVIATIONS CHG = chlorhexidine gluconate; $\mathrm{PVI}=$ povidone-iodine; $\mathrm{SSI}$ = surgical site infection.

SUBMITTED February 2, 2017. ACCEPTED May 24, 2017.

INCLUDE WHEN CITING Published online November 24, 2017; DOI: 10.3171/2017.5.SPINE17158. 
and anatomical locations containing different bacterial flora all contribute to the variability in surgeon preference. ${ }^{29}$ Respondents to one nationwide survey of 98 US academic neurosurgery programs showed a preference for the use of alcohol-based skin preparations..$^{15}$ On their choice for preoperative skin prophylaxis in spine surgeries, $57 \%, 53 \%$, and $38 \%$ of respondents had used DuraPrep surgical solution (iodine povacrylex and isopropyl alcohol), ChloraPrep (2\% chlorhexidine gluconate [CHG] and $70 \%$ isopropyl alcohol), and Betadine (7.5\% povidone-iodine [PVI] solution), respectively. Some centers use more than one skin prep in succession prior to each surgery, explaining why the total of the listed percentages does not add up to $100 \% .^{15}$

Two meta-analyses, neither of which is specific to spinal surgery, suggest a benefit from chlorhexidine over iodine-based preoperative skin antisepsis. ${ }^{16,21}$ Noorani et al. identified 6 studies with 5031 patients, finding a significant benefit with chlorhexidine $(\mathrm{p}=0.019){ }^{21}$ Lee et al. identified 9 randomized studies with 3614 patients, also finding a significant benefit with chlorhexidine use. ${ }^{16}$ Further analysis by Lee and colleagues revealed 4 separate randomized controlled trials that used positive skin cultures as the primary outcome measure for infection. . $^{6,10,24,28}$ Positive results with chlorhexidine ${ }^{16}$ have occurred in surgeries ranging from foot, ankle, ${ }^{6}$ and shoulder ${ }^{28}$ procedures to hysterectomies ${ }^{10}{ }^{1}$ suggesting a degree of versatility.

Worthy of particular mention is the study by Darouiche et al., ${ }^{11}$ a prospective, blinded, randomized controlled comparison of chlorhexidine-alcohol scrub to PVI scrub and paint in adults undergoing clean-contaminated general surgery procedures, with the primary outcome defined as SSI within 30 days of surgery. These authors found chlorhexidine to be significantly more protective against SSI $(p=0.008)$ in the 849 patients studied in both the intention-to-treat and per-protocol analyses. A significantly longer time to SSI was also noted in the chlorhexidine cohort, and no significant difference in adverse events was encountered.

We performed a prospective analysis of the use of ChloraPrep compared with Betadine for preoperative skin antisepsis in the prevention of SSI after spine surgery.

\section{Methods}

A prospective database was maintained for all spine operations performed by an academic neurosurgical department across 2 hospitals in the period from July 1, 2011, through August 31, 2015, for the purpose of identifying postoperative SSI in adult patients. Institutional review board approval was obtained prior to the start of this observational study period. Before instituting this study, we standardized factors that might influence the infection rate, including basing the type of preoperative antibiotics on institutional protocols for patient allergy status, patient weight, and procedure duration; ensuring antibiotics were received prior to incision; and making mandatory reports of any infections at the departmental level.

\section{Inclusion and Exclusion Criteria}

All consecutive patients who were undergoing a spinal operation and were older than 18 years of age were included in the study. Spinal surgeons in our neurosurgical department perform operations at 2 facilities in an academic campus, defined as facilities 1 and 2 for this study. The primary outcome measure was the occurrence of a spinal SSI. Surgical site infection was consistently defined throughout the study period by the need for incisional drainage, wound debridement, and irrigation in a patient with any history of spinal surgery at that particular spinal level.

Pediatric patients, defined as those younger than 18 years of age, were not included in the database because of the different facilities, surgeons, and infection protocols used for pediatric spinal care. Primary spinal infections were excluded as well.

\section{Preoperative Surgical Skin Antisepsis}

From July 1, 2011, through August 31, 2013, Betadine was consistently used as preoperative skin prophylaxis for all spinal operations, either anterior or posterior, and for all areas in the occipital, cervical, thoracic, lumbar, and sacral regions. From September 1, 2013, through December 31, 2015, both facilities 1 and 2 uniformly switched to the use of ChloraPrep for all spinal procedures. The minimum follow-up period was set at 12 months. Patients unable to return for a clinic visit were evaluated by electronic correspondence.

\section{Skin Disinfectant Protocol}

All patients underwent pretreatment of the skin with $70 \%$ isopropyl alcohol to an agreed upon perimeter established by the attending physician. With a sterile technique, Betadine scrub was applied thoroughly for 3 minutes using 2 large, winged PVI scrub sponges (PVI 7.5\%, Aplicare Inc.), and then pat dry with a sterile towel. Eight milliliters of Betadine solution (PVI 10\%) was then applied to sterile sponge sticks and painted over the desired prep field and allowed to completely dry, for a minimum of 3 minutes. For ChloraPrep use (CareFusion Inc.), 2 large 26$\mathrm{ml}$ applicators were applied to the skin in a sterile fashion after pretreatment of the skin with isopropyl alcohol in the manner described above, with a minimum drying time of 3 minutes. The patients were instructed to shower using a chlorhexidine body wash the night before (Hibiclens scrub, 4\% CHG, Mölnlycke Health Care).

\section{End Point Measures}

Evidence supporting a spinal infection can vary among individual patients. The majority of patients presented with fever, malaise, wound drainage and dehiscence, elevated erythrocyte sedimentation rate, C-reactive protein, and positive intraoperative cultures. The primary end point measure was the frequency of SSI, defined as spinal reoperation at the same surgical location for incisional drainage, debridement, and irrigation, as well as the incidence of SSI despite the use of Betadine or ChloraPrep. All patients requiring operative debridement and the administration of intravenous antibiotics were included to ensure consistency of SSI diagnosis during the study period. Deep infections were treated with incision and drainage 
of the wound, debridement, pulse lavage, and maintenance of spinal instrumentation. ${ }^{18}$ In cases of chronic infection or instrumentation loosening, the hardware was removed. Superficial skin infections and stitch granulomas that did not require admission and/or operative debridement were excluded.

For all patients meeting the diagnosis of SSI, the choice of Betadine or ChloraPrep was recorded. Other noted variables included the primary surgeon, patient age, surgical indication for the index case performed at this institution, the presence or absence of spinal instrumentation, and the microorganism cultured from intraoperative wound cultures. Total case volume and the rate of infection were stratified by each surgeon for analysis. A revision operation was defined as an operation performed at the same spinal level as a prior surgery regardless of the choice of prior approach. Surgical indications were classified as degenerative, scoliosis, trauma, tumor, and other. Operative reports were reviewed for cases that were "minimally invasive" and included those with endoscopic assistance, tubular decompression, mini-open lateral approaches, and percutaneous pedicle screw placement. ${ }^{19}$ It is well recognized that surgeries that are muscle sparing result in a reduced (possibly 10 -fold) postoperative infection rate. ${ }^{22}$ Minimally invasive approaches run the gamut but in this article include tubular, endoscopic, and muscle-splitting mini-open retroperitoneal approaches and percutaneous muscle-sparing instrumentation.

\section{Power Analysis}

Sample size calculations were based on infection rates during spinal surgery according to our own deep spinal infection rates in the 2 years prior to the start of the study. We estimated a $2 \%$ risk of SSI in patients undergoing skin preparation with Betadine, as previously reported by $\mathrm{Da}-$ rouiche et al. ${ }^{11}$ With a power of $80 \%$ and an alpha of 0.05 , conservative analysis based on equivalence 2 -sided power analysis revealed that 6000 patients would have to be enrolled to have a $45 \%$ decrease in the SSI risk between patients receiving Betadine and those receiving ChloraPrep.

\section{Statistical Analysis}

Continuous variables are presented as the mean \pm standard deviation, and categorical variables are presented as the frequency and percent. Analyses were performed using the Wilcoxon rank-sum, chi-square, and Fisher exact tests, as appropriate. A p value $\leq 0.05$ was considered statistically significant. Statistical analysis was performed using Stata 14.0 (StataCorp LLC).

\section{Results}

A total of 6959 consecutive spinal surgery patients (mean age $59.3 \pm 15.4$ years) were identified from July 1 , 2011, through August 31, 2015. In the Betadine and ChloraPrep cohorts, there were 3185 (45.8\%) and 3774 (54.2\%) patients, respectively. There were $1775(25.5 \%)$ and 1231 (17.7\%) female patients in the Betadine and ChloraPrep cohorts, respectively ( $\mathrm{p}<0.001$; Table 1$)$. In the ChloraPrep cohort, there was a significantly higher proportion of less invasive procedures $(7.04 \%$ vs $5.67 \%$, p < 0.001$)$ and
TABLE 1. Background characteristics in 6959 spinal surgery patients

\begin{tabular}{lrrr}
\hline \multicolumn{1}{c}{ Characteristic } & \multicolumn{1}{c}{ Betadine } & ChloraPrep & p Value \\
\hline Total & $3185(45.8 \%)$ & $3774(54.2 \%)$ & 0.274 \\
\hline Female & $1775(25.5 \%)$ & $1231(17.7 \%)$ & $<0.001$ \\
\hline Mean age in yrs \pm SD & $59.7 \pm 15.5$ & $58.6 \pm 15.4$ & 0.003 \\
\hline Disease & & & $<0.001$ \\
\hline Degenerative & $2286(32.8 \%)$ & $2732(39.2 \%)$ & 0.248 \\
\hline Scoliosis & $117(1.68 \%)$ & $108(1.55 \%)$ & 0.207 \\
\hline Tumor & $288(4.14 \%)$ & $422(6.06 \%)$ & 0.208 \\
\hline Trauma/fracture & $215(3.09 \%)$ & $103(1.48 \%)$ & 0.211 \\
\hline Other & $279(4.01 \%)$ & $409(5.88 \%)$ & 0.208 \\
\hline Open approach & $2790(40.1 \%)$ & $3284(47.2 \%)$ & $<0.001$ \\
\hline Less invasive approach & $395(5.68 \%)$ & $490(7.04 \%)$ & $<0.001$ \\
\hline Arthrodesis & $1318(18.9 \%)$ & $1337(19.2 \%)$ & 0.751 \\
\hline Revision index case & $197(2.83 \%)$ & $361(5.19 \%)$ & $<0.001$ \\
\hline
\end{tabular}

Values expressed as the number of patients (\%) unless indicated otherwise. All percentages are based on the total number of patients included in the study.

revision procedures $(5.19 \%$ vs $2.83 \%, \mathrm{p}<0.001)$. Sixtynine SSIs were observed (mean patient age $59.0 \pm 15.0$ years), and there was no significant difference in the incidence of SSI in the patients prepared with Betadine (33 [1.036\%] of 3185) versus those prepared with ChloraPrep (36 [0.954\%] of 3774; $\mathrm{p}=0.728$; Table 2). Among the SSI cases, 67 (97\%) had been performed via an open approach and $2(3 \%)$ via minimally invasive operations. Among the 67 open procedures, there were $31(46 \%)$ and $36(54 \%)$ cases of SSI in the Betadine and ChloraPrep cohorts, respectively. The incidence of SSI for minimally invasive and open surgery was $0.226 \%$ ( 2 of 885 cases) and $1.103 \%$ (67 of 6074 cases), respectively. Among the patients with SSI, the most common indication was degenerative dis-

TABLE 2. Surgical site infection: characteristics by skin antisepsis agent

\begin{tabular}{lccc}
\hline \multicolumn{1}{c}{ Characteristic } & Betadine & ChloraPrep & p Value \\
\hline Total patients & 3185 & 3774 & 0.274 \\
\hline No. of SSls & $33(1.036 \%)$ & $36(0.954 \%)$ & 0.728 \\
\hline Mean age in yrs \pm SD & $54 \pm 13.84$ & $62 \pm 14.59$ & \\
\hline Disease & & & \\
\hline Degenerative & $23(69.7 \%)$ & $25(69.4 \%)$ & 0.355 \\
\hline Scoliosis & $2(6.06 \%)$ & $5(13.9 \%)$ & 0.202 \\
\hline Tumor & $3(9.09 \%)$ & $4(11.1 \%)$ & 0.214 \\
\hline Trauma & $3(9.09 \%)$ & 0 & 0.228 \\
\hline Other & $2(6.06 \%)$ & $2(5.56 \%)$ & 0.213 \\
\hline Instrumentation & $21(63.6 \%)$ & $30(83.3 \%)$ & 0.308 \\
\hline Revision surgery & $18(54.5 \%)$ & $20(55.6 \%)$ & 0.306 \\
\hline Less invasive procedure & $1(3.03 \%)$ & $1(2.78 \%)$ & 0.209 \\
\hline Open procedure & $31(93.9 \%)$ & $36(100 \%)$ & 0.462 \\
\hline Al percentages based onte &
\end{tabular}

All percentages based on the total for each infection cohort (Betadine $=33$ cases, ChloraPrep $=36$ cases), except for SSI, which is based on the total for each antisepsis group. 
ease (48 [69.6\%] of 69). Fifty-one (74\%) patients with SSI had undergone instrumented fusions in the index operation, and 38 (55\%) patients with SSI had undergone revision surgeries. The patients treated at facilities 1 and 2 numbered 4495 (64.6\%) and 2464 (35.4\%), respectively, and there was no significant difference in the incidence of infection in the patients treated at facility 1 (52 [1.157\%] of 4495) versus facility $2(17$ [0.690\%] of 2464; $p=0.06)$. Seven primary surgeons performed all cases, with the incidence of SSI ranging from $0 \%$ to $1.846 \%$ (Table 3). Grampositive organisms were the most commonly cultured after surgery ( 45 [71.4\%] of 63 positive culture swabs; Table 4). In 11 cases (15.9\%), the intraoperative cultures were negative. The rates of SSI varied significantly among the 7 spine surgeons $(\mathrm{p}=0.011)$.

\section{Discussion}

Surgical site infection will continue to be a topic of focus in future studies as payers for these complications continue to decline or, as is the case with Medicare and Medicaid, when payments cease altogether. ${ }^{1}$ Surgical site antisepsis is just one part of a long sequence of steps that could play a role in the incidence of SSI. While the comparison of CHG to PVI has been performed in various surgical disciplines, such as general surgery, foot and ankle surgery, shoulder surgery, and plastic surgery, spinal surgery remains an area of limited study in a growing field. . $^{16,21,23,28,31,33}$ In 2012 in a prospective randomized study of 100 patients undergoing lumbar spinal surgery, Savage and colleagues evaluated the use of CHG compared with DuraPrep in terms of the ability of these agents to eliminate bacterial flora from the lumbar surgical site. ${ }^{29}$ No significant difference in efficacy was found between the two, although the incidence of postoperative SSI was not tracked.

When applying the current study criteria for detecting a clinically significant difference in SSI, many of the previously published studies are underpowered to detect the small clinical difference expected when specifically evaluating the efficacy of skin antisepsis on SSI. There is also a lack of agreement regarding what constitutes a clinically relevant difference. Two recent randomized con-

TABLE 3. Primary surgeon and case volume, type, and SSI

\begin{tabular}{lrrcrc}
\hline \multicolumn{1}{c}{ Parameter } & Total & MIS & $\begin{array}{c}\text { Open } \\
\text { Procedure }\end{array}$ & SSI & $\begin{array}{c}\text { SSI Incidence } \\
(\%)\end{array}$ \\
\hline No. of cases & 6959 & 885 & 6074 & 69 & \\
\hline Primary surgeon & & & & & \\
\hline 1 & 1504 & 422 & 1082 & 9 & 0.598 \\
\hline 2 & 1326 & 0 & 1326 & 12 & 0.905 \\
\hline 3 & 1134 & 111 & 1023 & 6 & 0.529 \\
\hline 4 & 1337 & 253 & 1084 & 18 & 1.346 \\
\hline 5 & 921 & 43 & 878 & 17 & 1.846 \\
\hline 6 & 597 & 27 & 570 & 7 & 1.173 \\
\hline 7 & 140 & 29 & 111 & 0 & 0.000 \\
\hline$p$ value & 0.002 & 0.003 & 0.008 & - & 0.011 \\
\hline
\end{tabular}

MIS = minimally invasive surgery.
TABLE 4. Most common microbiology findings of intraoperative cultures in SSI cases

\begin{tabular}{lcc}
\hline \multirow{2}{*}{ Organism } & \multicolumn{2}{c}{ No. of Cases } \\
\cline { 2 - 3 } & Betadine & ChloraPrep \\
\hline Methicillin-resistant Staphylococcus aureus & 9 & 8 \\
\hline Methicillin-sensitive S. aureus & 6 & 8 \\
\hline Coagulase-negative Staphylococcus spp. & 6 & 8 \\
\hline Corynebacterium spp. & 1 & 2 \\
\hline Gram-negative organisms & 5 & 8 \\
\hline Negative culture & 5 & 6 \\
\hline Acid-fast-positive organisms & 0 & 1 \\
\hline Polymicrobial cultures & 2 & 4 \\
\hline
\end{tabular}

trolled studies were designed with the intention of detecting differences in SSI. Swenson and associates compared DuraPrep, ChloraPrep, and Betadine in 3209 operations, finding SSI rates of $3.9 \%, 7.1 \%$, and $6.4 \%$, respectively (p $=0.002) .{ }^{31}$ Darouiche and coauthors evaluated 849 patients to compare ChloraPrep with PVI scrub in general surgery procedures and found significantly less superficial $(4.2 \%$ vs $8.6 \%, p=0.008)$ and deep $(1 \%$ vs $3 \%, p=0.05)$ infections in the ChloraPrep group. ${ }^{11}$ However, several key differences should be noted. Surgical site infection surveillance in both studies was limited to 30 days postoperatively, thus not capturing many indolent organisms, such as Propionibacterium spp., which has been shown to persist in the skin immediately following preoperative skin antisepsis with ChloraPrep and can take longer than 30 days to manifest clinically. ${ }^{16,17,21}$ Moreover, the translational value of study findings from contaminated or cleancontaminated operations using different study populations is questionable. Furthermore, the site of surgery mattersvariability in the bacterial flora can contribute to significant bias, especially in those studies with insufficiently small study populations.

In the present study, we prospectively followed 6959 consecutive spinal surgery patients, finding no significant difference in the incidence of SSI regardless of the choice of Betadine or ChloraPrep. Neither was there a significant difference in the incidence of SSI between the 2 study facilities $(1.157 \%$ and $0.690 \%$ in facilities 1 and 2, respectively; $\mathrm{p}=0.060$ ). This consistency held across 2 major facilities despite small variations in patient populations, and the procedures performed (Table 1) validate our results. Each facility contained a study population (4495 and 2464 patients, respectively) rivaling those in 2 of the largest studies of preoperative skin antisepsis in the literature. ${ }^{16,21}$ Another strength of our study is our strict adherence to the criteria for defining SSI. We defined a need to return to the operating room for incision and debridement as an SSI. Sixty-three $(91 \%)$ patients who returned to the operating room had positive intraoperative cultures. With this simple criterion, the need to interpret the heterogeneity in the preoperative diagnostic workup consisting of labs, radiological imaging, and recent antibiotic use was obviated. Therefore, 6 patients with purulent drainage could be included in the estimated SSI rate. Our data could help to guide future spine surgeons in their practice as signifi- 
cant disparity exists today among North American spine surgeons regarding the choice of skin antisepsis agent and there are currently no established guidelines for SSI prevention after open surgery. ${ }^{15}$

The lack of a significant difference in the incidence of SSI between Betadine and ChloraPrep is useful in clinical scenarios in which a surgeon may have reservations about the use of Betadine, such as in a patient with an iodine allergy. However, while chlorhexidine agents have been demonstrated to have an immediate onset of action, a relatively longer duration of coverage, and excellent coverage of both gram-positive and -negative bacteria, yeasts, and some viruses, this versatility comes at a relatively higher financial cost than that of iodine-based compounds. ${ }^{16}$ In a 2010 estimate of commercial costs, ChloraPrep, DuraPrep, and PVI were listed at \$708, \$427, and \$171 (US dollars) per 100 surgeries. ${ }^{36}$ In a systematic review and cost analysis of chlorhexidine versus iodine, Lee et al. found a cost benefit with chlorhexidine use ${ }^{16}$ However, this conclusion was based on the assumption that studies of dissimilar populations and designs are ultimately generalizable.

One additional concern with the use of alcohol-based preparations such as ChloraPrep is the risk of fire from electrocautery when the skin preparation is not allowed to fully dry, which requires at least 3 minutes. ${ }^{5,7,26}$ Corneal damage due to the agent's toxicity has been reported. Prone cervical spine surgery is a situation in which chlorhexidine can leak into the eye and go unmonitored. A few cases of corneal damage have resulted in various degrees of vision loss up to blindness, some even requiring corneal transplants after the use of chlorhexidine. ${ }^{32}$ The relative neurotoxicity of chlorhexidine to iodine agents has been demonstrated by in vitro studies comparing Schwann and neuroblastoma cell viability after exposure to varying concentrations of chlorhexidine and PVI..$^{3}$ One prevailing concern is the potential harm of chlorhexidine when the meninges and neural tissue are exposed to it. Sviggum et al. retrospectively reviewed 11,095 patients who had received 12,465 spinal anesthetics and chlorhexidine to determine if chlorhexidine contact with the meninges via a percutaneous route was clinically significant. ${ }^{30}$ Since the rate of neurological deficit was very low in their study $(0.04 \%)$, the authors were unable to show any added neurological risk. Note that similar rates have been published by Auroy et al. $(0.03 \%-0.06 \%),{ }^{3,4}$ The clinical concern in spinal surgery applications with a potentially neurotoxic antiseptic agent prior to skin incision with chlorhexidine is limited to cases beginning with open spinal wounds, a scenario lacking data in human subjects. Betadine, however, can be safely used around the dura and paraspinal soft tissues, mucous membranes, and other sensitive tissues, such as the conjunctiva and cornea. ${ }^{8,15,27}$ Corneal exposure to chlorhexidine cannot be allowed to happen at the concentrations used for skin sterilization. ${ }^{32}$ The standard concentration used in ophthalmological procedures is often much lower, for example, $0.02 \%$ in one study of antisepsis in cataract procedures. ${ }^{14}$

Relative weaknesses of the current study include a lack of blinding and randomization. Multiple patient, disease, and surgery-specific risk factors for infection have been identified in posterior spinal surgery and could potentially bias our study. A univariate analysis of all available factors was performed; however, multivariate logistic regression analysis could not be performed because of the limited subgroup sizes.

A growing number of patient, disease, and surgery-specific variables associated with the development of SSIs are being identified in the literature. Most recently, in a retrospective study of 12,528 cranial and spinal surgeries, Wathen et al. correlated SSI with operating room personnel turnover ${ }^{34}$ Despite variations in baseline characteristics, we believe that our study data are generalizable because of a strict institutional adherence to preoperative antisepsis across a large number of consecutive patients encompassing a variety of spinal pathologies.

\section{Conclusions}

The choice of either ChloraPrep or Betadine for preoperative skin antisepsis in spinal surgery did not significantly impact the rate of postoperative SSI.

\section{References}

1. Anderson DJ, Hartwig MG, Pappas T, Sexton DJ, Kanafani ZA, Auten G, et al: Surgical volume and the risk of surgical site infection in community hospitals: size matters. Ann Surg 247:343-349, 2008

2. Astagneau P, Rioux C, Golliot F, Brücker G: Morbidity and mortality associated with surgical site infections: results from the 1997-1999 INCISO surveillance. J Hosp Infect 48:267-274, 2001

3. Auroy Y, Benhamou D, Bargues L, Ecoffey C, Falissard B, Mercier FJ, et al: Major complications of regional anesthesia in France: the SOS Regional Anesthesia Hotline Service. Anesthesiology 97:1274-1280, 2002 (Erratum in Anesthesiology 98:595, 2003)

4. Auroy Y, Narchi P, Messiah A, Litt L, Rouvier B, Samii K: Serious complications related to regional anesthesia: results of a prospective survey in France. Anesthesiology 87:479486, 1997

5. Barker SJ, Polson JS: Fire in the operating room: a case report and laboratory study. Anesth Analg 93:960-965, 2001

6. Bibbo C, Patel DV, Gehrmann RM, Lin SS: Chlorhexidine provides superior skin decontamination in foot and ankle surgery: a prospective randomized study. Clin Orthop Relat Res 438:204-208, 2005

7. Briscoe CE, Hill DW, Payne JP: Inflammable antiseptics and theatre fires. Br J Surg 63:981-983, 1976

8. Cheng MT, Chang MC, Wang ST, Yu WK, Liu CL, Chen TH: Efficacy of dilute betadine solution irrigation in the prevention of postoperative infection of spinal surgery. Spine (Phila Pa 1976) 30:1689-1693, 2005

9. Coello R, Charlett A, Wilson J, Ward V, Pearson A, Borriello P: Adverse impact of surgical site infections in English hospitals. J Hosp Infect 60:93-103, 2005

10. Culligan PJ, Kubik K, Murphy M, Blackwell L, Snyder J: A randomized trial that compared povidone iodine and chlorhexidine as antiseptics for vaginal hysterectomy. Am J Obstet Gynecol 192:422-425, 2005

11. Darouiche RO, Wall MJ Jr, Itani KM, Otterson MF, Webb AL, Carrick MM, et al: Chlorhexidine-alcohol versus povidone-iodine for surgical-site antisepsis. N Engl J Med 362:18-26, 2010

12. Dimick JB, Chen SL, Taheri PA, Henderson WG, Khuri SF, Campbell DA Jr: Hospital costs associated with surgical complications: a report from the private-sector National 
Surgical Quality Improvement Program. J Am Coll Surg 199:531-537, 2004

13. Doan L, Piskoun B, Rosenberg AD, Blanck TJ, Phillips MS, Xu F: In vitro antiseptic effects on viability of neuronal and Schwann cells. Reg Anesth Pain Med 37:131-138, 2012

14. Inagaki K, Yamaguchi T, Ohde S, Deshpande GA, Kakinoki $\mathrm{K}$, Ohkoshi K: Bacterial culture after three sterilization methods for cataract surgery. Jpn J Ophthalmol 57:74-79, 2013

15. Kalb S LR, Dididze M, Gaynor B, Wang MY, Levi AD: The use of anti-septic solutions in the prevention of neurosurgical site infections. Clin Microbiol 2:124, 2013

16. Lee I, Agarwal RK, Lee BY, Fishman NO, Umscheid CA: Systematic review and cost analysis comparing use of chlorhexidine with use of iodine for preoperative skin antisepsis to prevent surgical site infection. Infect Control Hosp Epidemiol 31:1219-1229, 2010

17. Lee MJ, Pottinger PS, Butler-Wu S, Bumgarner RE, Russ SM, Matsen FA III: Propionibacterium persists in the skin despite standard surgical preparation. J Bone Joint Surg Am 96:1447-1450, 2014

18. Levi AD, Dickman CA, Sonntag VK: Management of postoperative infections after spinal instrumentation. J Neurosurg 86:975-980, 1997

19. McAfee PC, Garfin SR, Rodgers WB, Allen RT, Phillips F, Kim C: An attempt at clinically defining and assessing minimally invasive surgery compared with traditional "open" spinal surgery. SAS J 5:125-130, 2011

20. Mooney V, Bernard JA, Friedman MM, Letz G: Symposium on cost containment issues in spine surgery. Spine (Phila Pa 1976) 19:1867-1871, 1994

21. Noorani A, Rabey N, Walsh SR, Davies RJ: Systematic review and meta-analysis of preoperative antisepsis with chlorhexidine versus povidone-iodine in clean-contaminated surgery. Br J Surg 97:1614-1620, 2010

22. O'Toole JE, Eichholz KM, Fessler RG: Surgical site infection rates after minimally invasive spinal surgery. J Neurosurg Spine 11:471-476, 2009

23. Ostrander RV, Botte MJ, Brage ME: Efficacy of surgical preparation solutions in foot and ankle surgery. J Bone Joint Surg Am 87:980-985, 2005

24. Paocharoen V, Mingmalairak C, Apisarnthanarak A: Comparison of surgical wound infection after preoperative skin preparation with $4 \%$ chlohexidine and povidone iodine: a prospective randomized trial. J Med Assoc Thai 92:898902, 2009

25. Pawar AY, Biswas SK: Postoperative spine infections. Asian Spine J 10:176-183, 2016

26. Prasad R, Quezado Z, St Andre A, O'Grady NP: Fires in the operating room and intensive care unit: awareness is the key to prevention. Anesth Analg 102:172-174, 2006

27. Rahn R: Review presentation on povidone-iodine antisepsis in the oral cavity. Postgrad Med J 69 (Suppl 3):S4-S9, 1993

28. Saltzman MD, Nuber GW, Gryzlo SM, Marecek GS, Koh JL: Efficacy of surgical preparation solutions in shoulder surgery. J Bone Joint Surg Am 91:1949-1953, 2009

29. Savage JW, Weatherford BM, Sugrue PA, Nolden MT, Liu
JC, Song JK, et al: Efficacy of surgical preparation solutions in lumbar spine surgery. J Bone Joint Surg Am 94:490-494, 2012

30. Sviggum HP, Jacob AK, Arendt KW, Mauermann ML, Horlocker TT, Hebl JR: Neurologic complications after chlorhexidine antisepsis for spinal anesthesia. Reg Anesth Pain Med 37:139-144, 2012

31. Swenson BR, Hedrick TL, Metzger R, Bonatti H, Pruett TL, Sawyer RG: Effects of preoperative skin preparation on postoperative wound infection rates: a prospective study of 3 skin preparation protocols. Infect Control Hosp Epidemiol 30:964-971, 2009

32. Tabor E, Bostwick DC, Evans CC: Corneal damage due to eye contact with chlorhexidine gluconate. JAMA 261:557558,1989

33. Veiga DF, Damasceno CA, Veiga-Filho J, Figueiras RG, Vieira RB, Florenzano FH, et al: Povidone iodine versus chlorhexidine in skin antisepsis before elective plastic surgery procedures: a randomized controlled trial. Plast Reconstr Surg 122:170e-171e, 2008

34. Wathen C, Kshettry VR, Krishnaney A, Gordon SM, Fraser $\mathrm{T}$, Benzel EC, et al: The association between operating room personnel and turnover with surgical site infection in more than 12,000 neurosurgical cases. Neurosurgery 79:889-894, 2016

35. Weber WP, Zwahlen M, Reck S, Feder-Mengus C, Misteli H, Rosenthal R, et al: Economic burden of surgical site infections at a European university hospital. Infect Control Hosp Epidemiol 29:623-629, 2008

36. Zhou S, Carlson A: Budget impact analysis of three presurgical antisepsis protocols, presented at the Fifth Decennial International Conference on Healthcare-Associated Infections, Atlanta, 2010 (Poster) (http://multimedia.3m. $\mathrm{com} / \mathrm{mws} / \mathrm{media} / 702005 \mathrm{O} /$ budget-impact-analysis-of-skinantisepsis-protocols.pdf) [Accessed August 16, 2017]

\section{Disclosures}

Dr. Levi has received a teaching honorarium from Medtronic.

\section{Author Contributions}

Conception and design: Levi. Acquisition of data: Levi, Starke, Jimsheleishvili, Dididze. Analysis and interpretation of data: Levi, Starke, Jimsheleishvili, Dididze. Drafting the article: Levi, Ghobrial. Critically revising the article: Levi, Wang, Crandall. Reviewed submitted version of manuscript: Levi, Green, Manzano, Vanni, Starke, Crandall, Dididze. Statistical analysis: Levi, Starke. Administrative/technical/material support: Levi. Study supervision: Levi.

\section{Correspondence}

Allan D. Levi, Department of Neurological Surgery, University of Miami Miller School of Medicine, Lois Pope LIFE Center, 1095 NW 14th Terrace (D4-6), Miami, FL 33136. email: alevi@med. miami.edu. 